\title{
Power Fluctuation
}

National Cancer Institute

\section{Source}

National Cancer Institute. Power Fluctuation. NCI Thesaurus. Code C92096.

The device failed due to fluctuations within the power supply (e.g. transient power, power spike, power dip, or power sequencing). 